17. W.S. Chen and K.Y. Ku, Broadband design of a small nonsymmetric ground $\lambda / 4$ open slot antenna, Microwave J 50 (2007), 110-120.

18. W.S. Chen and K.Y. Ku, Band-rejected design of the printed open slot antenna for WLAN/WiMAX operation, IEEE Trans Antennas Propag 56 (2008), 1163-1169.

19. C.H. Wu and K.L. Wong, Hexa-band internal printed slot antenna for mobile phone application, Microwave Opt Technol Lett 50 (2008), 35-38.

20. K.L. Wong, Y.W. Chi, and S.Y. Tu, Internal multiband printed folded slot antenna for mobile phone application, Microwave Opt Technol Lett 49 (2007), 1833-1837.

21. P.L. Sun, H.K. Dai, and C.H. Huang, Dual band slot antenna with single feed line, U.S. Patent 6,677,909 B2, Jan. 2004.

22. C.M. Su, H.T. Chen, F.S. Chang, and K.L. Wong, Dual-band slot antenna for 2.4/5.2 GHz WLAN operation, Microwave Opt Technol Lett 35 (2002), 306-308.

23. Ansoft Corporation HFSS, Available at: http://www.ansoft.com/ products/hf/hfss/.

(C) 2010 Wiley Periodicals, Inc.

\section{SIMPLIFIED DESIGN OF MULTILAYERED SUBSTRATE-INTEGRATED WAVEGUIDE RIBLET-SAAD COUPLERS}

Vladimir A. Labay ${ }^{1}$ and Jens Bornemann ${ }^{2}$

${ }^{1}$ Department of Electrical and Computer Engineering,

Gonzaga University, Spokane, WA 99258

${ }^{2}$ Department of Electrical and Computer Engineering, University of

Victoria, Victoria, BC, V8W 3P6, Canada; Corresponding author:

j.bornemann@ieee.org

Received 27 July 2009

ABSTRACT: A simplified design procedure for dual-and triple-layered Riblet-Saad directional couplers in substrate-integrated waveguide (SIW) technology is presented. Emphasis is placed on a design that considers ease of fabrication and includes interface ports such as microstrip-to-SIW transitions with straight, bent, and mitered microstrip lines. Designs are performed in an HFSS environment, which is verified by comparison with an independent field-solver simulation. (C) 2010 Wiley Periodicals, Inc. Microwave Opt Technol Lett 52: 1142-1144, 2010; Published online in Wiley InterScience (www.interscience. wiley.com). DOI 10.1002/mop.25149

Key words: substrate-integrated waveguide; multilayered E-plane couplers; Riblet-Saad directional couplers

\section{INTRODUCTION}

Substrate-integrated waveguide (SIW) technology has found many applications in the millimeter-wave frequency range, for example $[1,2]$, because it presents a reasonable compromise between microstrip and rectangular-waveguide circuitry. One of the advantages for the design of SIW components is that after dispersion characteristics and via hole dimensions have been related to all-dielectric-filled rectangular waveguides [3], traditional waveguide design strategies, for example, [4], can be used. Moreover, tapers to microstrip technology [5] provide an interface to measurement equipment.

Because of its planar nature, SIW applications are preferably fabricated in H-plane circuitry. Filters, diplexers, power dividers, and H-plane couplers are some of the preferred components, for example, [1, 6-8]. To integrate this technology with two-dimensional antenna arrays [9], however, coupling between stacked SIW components must be facilitated. Attempts to incorporate multilayered SIW circuits for antenna feed networks have been made in $[8,10]$ in the form of an E-plane directional couplers.
This article presents simplified design guidelines for dualand triple-layered Riblet-Saad SIW E-plane couplers. The designs include tight coupling and interface ports with straight, bent, and mitered microstrip transitions.

\section{DESIGN}

Figure 1 shows an eight-section dual-layer [Fig. 1(a)] and two triple-layer [Figs. 1(b) and 1(c)] Riblet-Saad couplers in SIW technology with straight, bent, and mitered microstrip ports. Note that a coupling aperture is represented by a slot pair-one in longitudinal and the other in transverse direction.

For the design of the SIW components, the substrate material as well as the placement, dimensions, and shapes of via holes must be selected. These parameters are usually dictated by the fabrication process involved. We assume standard printed-circuit board facilities and select RT Duroid with $\varepsilon_{\mathrm{r}}=2.2$, height $b=$ $0.508 \mathrm{~mm}$, and metallization thickness $t=35 \mu \mathrm{m}$ as substrate
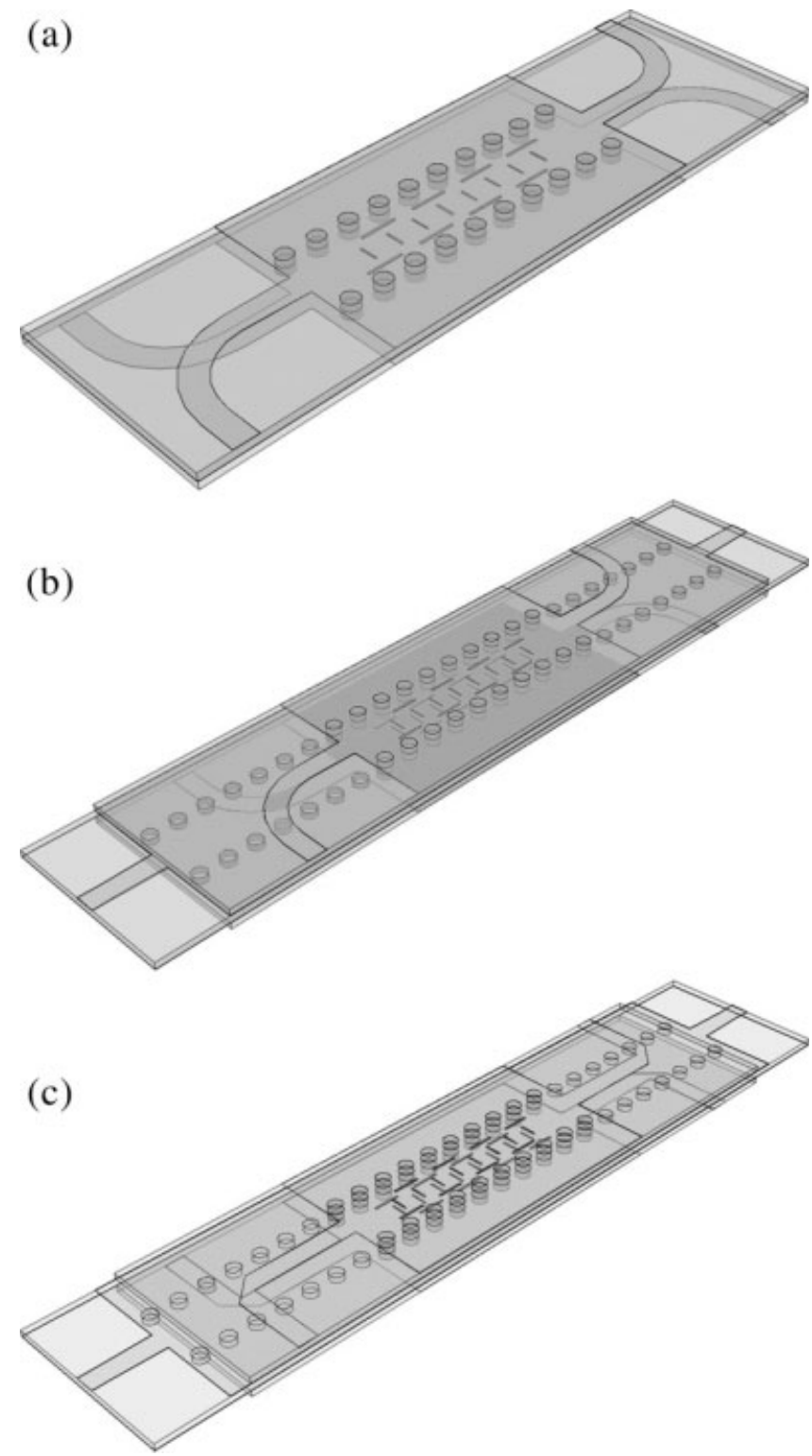

Figure 1 Eight-section Riblet-Saad couplers in SIW technology: (a) dual-layer with bent microstrip ports, (b) triple-layer with straight and bent microstrip ports, (c) triple-layer with straight and mitered microstrip ports 
material. We assume circular vias of diameter $2 r=1.19 \mathrm{~mm}$ and calculate their center-to-center spacings in width and length as $a=5.53 \mathrm{~mm}$ and $p=2.38 \mathrm{~mm}$, respectively, from closeform expressions in $[3,5]$. This leads to an equivalent all-dielectric-filled waveguide width of $a_{\text {equ }}=4.79 \mathrm{~mm}$ [5]. A transition from this SIW to microstrip can be obtained from [5]. The width of the $50-\Omega$ microstrip line is $1.67 \mathrm{~mm}$, the width at the SIW junction is $1.80 \mathrm{~mm}$, and the length of the linear taper between these two widths is $4.27 \mathrm{~mm}$. These dimensions are displayed in Figure 2. Bends and mitered corners are designed using standard procedures as outlined, for example, in [11].

The design process for the aperture dimensions follows, in principle, the equivalent-circuit synthesis approach of [4] and includes the aperture thickness $(2 t=70 \mu \mathrm{m})$ of the top and bottom metallization of the stacked SIWs. However, to avoid overlapping apertures for tight coupling and maintain ease of fabrication, all apertures in this simplified design are preset to be identical. For given specification and number of apertures, the equivalent-circuit parameters as obtained from [4] are averaged so that every aperture contributes the same amount of coupling. This leaves only a single aperture to be designed for which the design parameters are (c.f. Fig. 2): the lengths of the longitudinal $\left(l_{1}\right)$ and transverse $\left(l_{\mathrm{t}}\right)$ slots, and the distance $s$ from the center of the longitudinal slot to the centerline of the guide. The widths of the longitudinal $\left(w_{1}\right)$ and transverse $\left(w_{\mathrm{t}}\right)$ slots are set to standard end-mill cutter diameters of $0.154 \mathrm{~mm}$ and $0.1 \mathrm{~mm}$, respectively. Finally, all center-to-center distances $d$ between apertures are set to one quarter of the electrical length at the midband frequency. Parameter $d$ is the only dimension that is varied during a final one-dimensional optimization in HFSS to improve return loss and isolation.

Note that after an initial design is obtained following the earlier procedure, the coupling value usually deviates from its target by a few $\mathrm{dB}$. This is because of the fact that the fields are averaged over all slots to coincide with a single combined equivalent-circuit parameter. The initial design is thus repeated for a coupling value to offset the deviation obtained in the previous step. Triple-layer couplers are designed in the same way as dual-layer components but for an initially lower coupling value, considering the fact that coupling will double once the third layer is added.

\section{RESULTS}

The first design example is a 3-dB eight-section dual-layer Riblet-Saad coupler according to Figure 1(a). Figure 3 shows a

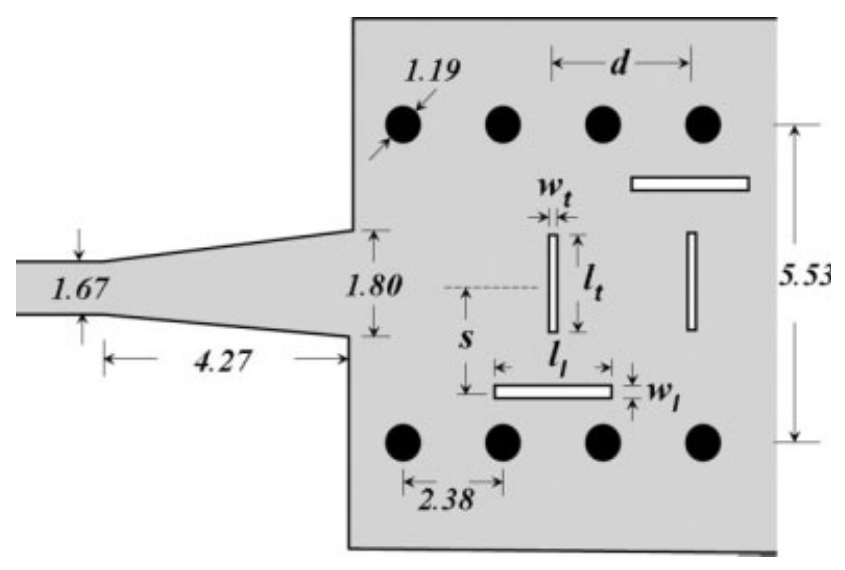

Figure 2 Top view and dimensions (in $\mathrm{mm}$ ) of SIW-to-microstrip transition and coupling slots as explained in Section 2
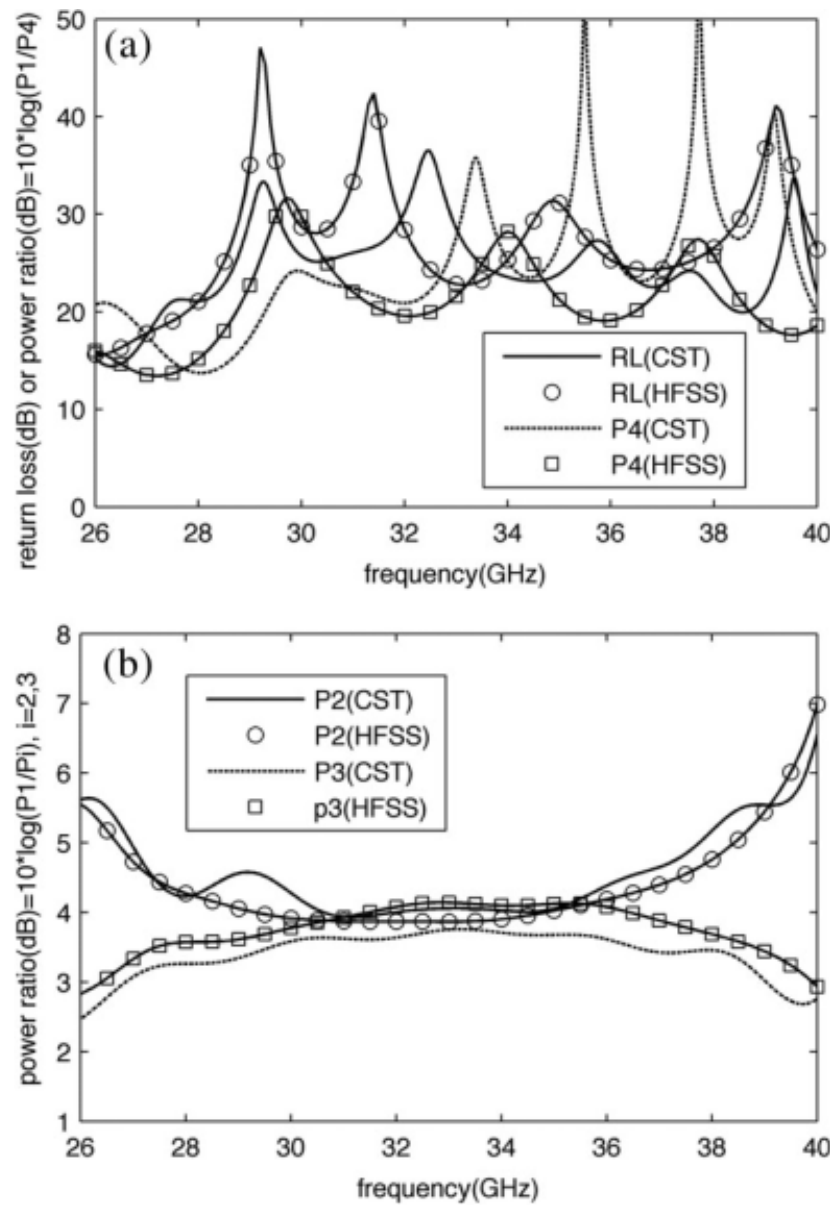

Figure 3 Dual-layer eight-section 3-dB Riblet-Saad coupler with microstrip-to-SIW transitions according to Figure 1(a): performance comparison between HFSS and CST for return loss and isolation (a) and performance comparison between HFSS and CST for through port and coupled port (b)

comparison between results obtained with HFSS and CST Microwave Studio. Port assignments are: input port (1), through port (2), coupled port (3), and isolated port (4). The comparisons between HFSS and CST for return loss and isolation [Fig. 3(a)] and for coupled and through ports [Fig. 3(b)] show good agreement, especially when considering that both field solvers include all four SIW-to-microstrip transitions. Figure 3, thus, validates the simplified design procedure as outlined in the previous section. A comparison with measurements obtained from a 3-dB SIW coupler with 14 dual-hole apertures (instead of the RibletSaad configurations presented here) has already been presented in [10] and will not be repeated.

As a second example, Figure 4 shows the performances of two triple-layer eight-section Riblet-Saad couplers. The design in Figure 4(a) features bent [Fig. 1(b)] microstrip connections, whereas that in Figure 4(b) uses mitered ones [Fig. 1(c)]. For this arrangement, the center layer operates as the input (1) and through port (2); coupled ports are (3) and (5), isolated ports (4) and (6). The couplers are designed for three-way power division, that is, $4.78 \mathrm{~dB}$, between the through port and the two coupled ports. It is clearly observed that the coupler with bent microstrip ports [Fig. 4(a)] achieves better return loss and isolation performance compared with the one with mitered sections [Fig. 

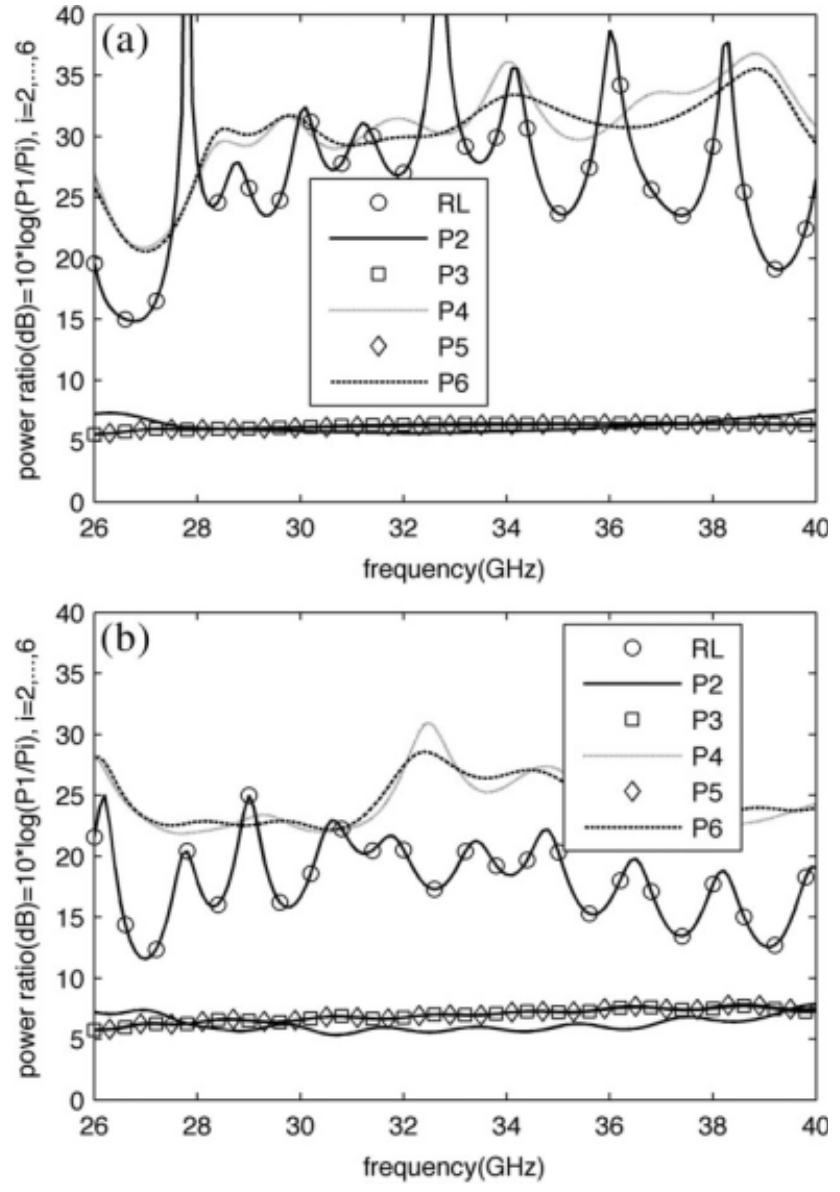

Figure 4 Performances of triple-layer eight-section Riblet-Saad 4.78$\mathrm{dB}$ couplers with (a) straight/bent microstrip ports according to Figure 1(b) and (b) straight/mitered microstrip ports according to Figure 1(c)

4(b)]. As a result, the power splitting performance in Figure 4(a) is fairly constant over almost the entire Ka-band.

For completeness, Table 1 presents the aperture dimensions of the two couplers presented in Figures 3 and 4. The remaining design parameters and nomenclature are as outlined in Figure 2 and Section 2.

\section{CONCLUSIONS}

The simplified design approach presents a viable option for multilayered coupler design in SIW technology. It contributes to simple fabrication due to keeping identical all aper- tures dimensions in a coupler and maintaining a constant distance between them. Dual- and triple-layered RibletSaad couplers with power divisions of $3 \mathrm{~dB}$ and $4.78 \mathrm{~dB}$ are presented as examples of multilayered SIW technology. The basic design procedure is performed in an HFSS environment and validated by comparison with results obtained with another commercially available software package. For access to all ports, microstrip-to-SIW transitions are included and are shown to influence the performance of the Riblet-Saad SIW couplers.

\section{REFERENCES}

1. E. Moldovan, R.G. Bosisio, and K. Wu, W-band multiport substrate-integrated waveguide circuits, IEEE Trans Microwave Theory Tech 54 (2006), 625-632.

2. D. Stephens, P.R. Young, and I.D. Robertson, Millimeter-wave substrate integrated waveguides and filters in photoimageable thick-film technology, IEEE Trans Microwave Theory Tech 53 (2005), 3832-3838.

3. Y. Cassivi, L. Perregrini, P. Arcioni, M. Bressan, K. Wu, and G. Conciauro, Dispersion characteristics of substrate integrated rectangular waveguide, IEEE Microwave Wireless Compon Lett 12 (2002), 333-335.

4. J. Uher, J. Bornemann, and U. Rosenberg, Waveguide components for antenna feed systems: Theory and CAD, Artech House Inc., Norwood, 1993.

5. D. Deslandes and $\mathrm{K}$. Wu, Integrated microstrip and rectangular waveguide in planar form, IEEE Microwave Wireless Compon Lett 11 (2001), 68-70.

6. E. Moldovan, R.G. Bosisio, and K. Wu, W-band multiport substrate-integrated waveguide circuits, IEEE Trans Microwave Theory Tech 54 (2006), 625-632.

7. H.J. Tang, W. Hong, J.-X. Chen, G.Q. Luo, and K. Wu, Development of millimeter-wave planar diplexers based on complementary characters of dual-mode substrate integrated waveguide filters with circular and elliptic cavities, IEEE Trans Microwave Theory Tech 55 (2007), 776-782.

8. Y. Cassivi, D. Deslandes, and K. Wu, Substrate integrated waveguide directional couplers, In: Proceedings of Asia-Pacific Microwave Conference, FR1B-3, Kyoto, Japan, November 2002, $4 \mathrm{p}$.

9. K. Kuhlmann, K. Rezer, and A.F. Jacob, Circularly polarized substrate-integrated waveguide antenna array at Ka-band, In: Proceedings of German Microwave Conference, Hamburg, Germany, March 2008, pp. 471-474.

10. V.A. Labay and J. Bornemann, E-Plane directional couplers in substrate-integrated waveguide technology, In: Proceedings 2008 AsiaPacific Microwave Conference, A1-75, Hong Kong, December 2008, 4 p.

11. K.C. Gupta, R. Garg, I. Bahl, and P. Bhartia, Microstrip lines and slotlines, 2nd ed., Artech House Inc., Norwood, 1996.

(c) 2010 Wiley Periodicals, Inc.

TABLE 1 Aperture Dimensions in $\mathrm{mm}$

\begin{tabular}{lcccc}
\hline $\begin{array}{l}\text { Coupling } \\
(\mathrm{dB})\end{array}$ & $\begin{array}{c}\text { Longitudinal } \\
\text { Slots }\end{array}$ & $\begin{array}{c}\text { Transverse } \\
\text { Slots }\end{array}$ & $\begin{array}{c}\text { Distance Between } \\
\text { Apertures }\end{array}$ & Figure \\
\hline 3 & $l_{1}=2.55$ & $l_{\mathrm{t}}=1.25$ & $d=1.98$ & 3 \\
& $w_{1}=0.154$ & $w_{\mathrm{t}}=0.10$ & & \\
& $s=1.37$ & & & \\
4.78 & $l_{1}=2.25$ & $l_{\mathrm{t}}=1.40$ & $d=1.98$ & 4 \\
& $w_{1}=0.154$ & $w_{\mathrm{t}}=0.10$ & & \\
& $s=1.37$ & & &
\end{tabular}

